\title{
Systemic therapy for patients with advanced, unresectable or metastatic renal cell carcinoma: moving to guidelines
}

\author{
Sébastien J. Hotte, MD; Anil K. Kapoor, MD ${ }^{\dagger}$
}

\begin{abstract}
Until recently, patients with advanced, unresectable or metastatic renal cell cancer (RCC) had very few therapeutic options. Cytokine therapy, consisting mainly of interferon- $\alpha$ and interleukin-2, was considered the mainstay of therapy. A better understanding of the biology of RCC has led to the development of novel therapeutic agents that target angiogenesis. Inactivation of the von Hippel-Lindau tumour-suppressor gene $V H L$, which is present in the vast majority of clear-cell RCC tumours, leads to overexpression of the vascular endothelial growth factor, which in turn promotes angiogenesis. Recent observations from a number of positive studies with agents such as sunitinib malate, sorafenib, temsirolimus and bevacizumab have led to a rapid and exciting change in the standard of care for patients with advanced renal cell carcinoma. This article reviews these agents in the context of their use in clinical practice and provides suggestions about the appropriateness of various agents in specific clinical situations.
\end{abstract}

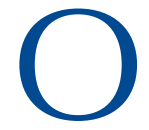
ver 4600 patients are diagnosed yearly with renal cell cancer (RCC) in Canada, and 1500 patients die from the disease. ${ }^{1}$ At the time of their first diagnosis, $45 \%$ of patients will have localized disease, $25 \%$ will have locally advanced disease with lymphnode or local organ involvement, and the remaining 30\% will have metastases. ${ }^{2}$ Patients with metastatic disease have a 5-year life expectancy of less than $10 \%$ and a median survival time of less than 12 months. However, survival can be quite variable; it depends on a number of prognostic factors, including performance status; lactate dehydrogenase, hemoglobin and calcium levels; and the absence of prior nephrectomy. ${ }^{3}$ Motzer and colleagues ${ }^{3}$ identified prognostic-risk categories based on the number of adverse risk factors present. These categories have been validated and used to stratify patients for clinical trials (i.e., 0 adverse prognostic factors $=$ favourable risk, 1 or $2=$ intermediate risk, and 3 or more = poor risk).

For patients who have inoperable or metastatic disease, cure is rarely possible; treatment efforts often centre on effectively controlling symptoms and offering a chance at improved survival. Clinical trials of chemotherapy for those with metastatic disease have shown that RCC is resistant to currently available chemotherapeutic agents. ${ }^{4}$ Immunotherapy agents, however, have shown activity in RCC (these are reviewed elsewhere in this supplement). For those with inoperable or metastatic RCC, interferon- $\alpha$ and interleukin-2 have been evaluated extensively with various doses and modes of delivery, and in com- bination with a number of cytotoxic agents. However, these cytokines are associated with only modest improvements in survival. Currently no universally accepted standard of care exists. In Canada, interferon has been considered the standard of care for patients with inoperable or metastatic RCC because of its modest survival, improvement when compared with that for noninterferon regimens and slightly more favourable toxicity profile than interleukin-2.

A growing understanding of the biology of RCC has led to the discovery of novel potential therapeutic targets. The von Hippel-Lindau tumour-suppressor gene $V H L$ was identified in 1993. ${ }^{5} \mathrm{VHL}$ inactivation, which is present in the vast majority of clear-cell RCC tumours, leads to overexpression of the vascular endothelial growth factor (VEGF), which in turn promotes angiogenesis and the formation of new blood vessels. ${ }^{5}$ VEGF is a tumour-secreted cytokine that plays an important role in both normal and tumour-associated angiogenesis, and exerts its effect through interaction with the transmembrane tyrosine-kinase receptors that are present on the cell surface (i.e., VEGF receptor [VEGFR]-1, -2, and -3$).{ }^{6}$ VEGFR-2 seems to be the main receptor responsible for the angiogenic effects of VEGF. (Molecular genetics, the role of $\mathrm{VHL}$ and the molecular characterization of RCC are all described in further detail elsewhere in this supplement.)

Anti-angiogenic therapy is the use of drugs or other substances to alter the flow of blood around or to a tumour. VEGF can be inhibited through a number of mechanisms. Agents such as bevacizumab target VEGF directly, whereas other small-molecule tyrosine-kinase inhibitors target receptors to VEGF and inhibit downstream cell signaling. Examples of these orally available compounds are sunitinib and sorafenib. 
The Genitourinary Cancer Disease Site Group of Cancer Care Ontario's Program in Evidencebased Care has recently been completing a systematic review of evidence from recent randomized controlled trials about angiogenesis inhibitors to better inform clinicians about which systemic therapy may be best suited to particular groups of patients (unpublished data, 2007). Although not meant to replace this systematic review, the current review draws on the information collected during the preparation of the Program in Evidencebased Care's guideline document to provide an overview of the role of inhibitors of angiogenesis in the systemic treatment of patients with advanced RCC. We discuss the evidence supporting the use of the agents for which there is the most evidence, namely sunitinib, sorafenib, bevacizumab and the mammalian target of rapamycin (mTOR)-inhibitor temsirolimus, in the clinical context of treatment for metastatic disease with first- or second-line therapy and for patients with a poor prognosis.

\section{Sunitinib malate}

Sunitinib malate is an oral inhibitor of a number of tyrosine kinases, including VEGFR and the platelet-derived growth factor receptor, ${ }^{7-9}$ that are known to play a significant role in the pathogenesis of RCC through their involvement with the $V H L$ gene. A phase 3 randomized trial ${ }^{10}$ recently published in the New England Journal of Medicine reported the superior efficacy of sunitinib malate over interferon for patients with locally advanced, unresectable or metastatic RCC who had had no previous systemic therapy. This study was based on the results of a pooled analysis of 2 phase 2 studies of sunitinib for patients who had undergone previous cytokine therapy and had a response rate of $42 \% .{ }^{11}$ In the phase 3 study, ${ }^{10}$ interferon was used as a comparator because of its wide use as a standard therapy for advanced RCC. Seven hundred and fifty patients were enrolled and randomized to receive either sunitinib (50 mg daily) for 28 days, followed by 14 days without treatment or interferon in escalating doses up to 9 million units subcutaneously 3 times weekly. The primary end point of the study was progression-free survival (PFS). Secondary end points included objective response rate, overall survival, patient-reported outcomes and safety. To be enrolled in the study, patients were required to have a clear-cell histological component and not to have received any previous systemic therapy for RCC. At the time of analysis, the median duration of treatment was 6 months in the sunitinib group and 4 months in the interferon group; treatment was ongoing for $66 \%$ of sunitinib patients and $34 \%$ of interferon patients.

Although sunitinib was well tolerated by most patients, most general adverse events of all grades occurred more frequently in the sunitinib group than in the interferon group. Thirty-eight percent of patients had a dose interruption because of adverse events, and $32 \%$ had a dose reduction in the sunitinib arm, compared with $32 \%$ and $21 \%$, respectively, for patients in the interferon arm. Relatively few patients experienced grade 3 or 4 adverse events, but these were more commonly observed in the sunitinib group than in the interferon group (12\% v. $7 \%$ ). Grade 3 diarrhea ( $4 \%$ v. $0 \%)$, vomiting ( $4 \%$ v. $1 \%)$, hypertension ( $8 \%$ v. $1 \%)$ and hand-foot syndrome (5\% v. $0 \%$ ) were experienced more often in the sunitinib group than in the interferon group. Typical effects of interferon, such as pyrexia, chills, myalgia and flu-like symptoms, were seen more often in patients receiving interferon than in those receiving sunitinib. The incidence of a grade 3 decline in the left ventricular ejection fraction was similar for both groups ( $2 \%$ v. $1 \%$ in the sunitinib and interferon groups, respectively). This decline was not associated with clinical consequences and was reversible when the treatment was discontinued or the dose was modified. Bone-marrow suppression was observed more often for patients receiving sunitinib than for those receiving interferon. Grade 3 or 4 leucopenia ( $5 \%$ of patients), neutropenia ( $12 \%$ of patients) and thrombocytopenia ( $8 \%$ of patients) were observed in sunitinib-treated patients. Two patients treated with sunitinib had an episode of febrile neutropenia.

All events were assessed by blinded central review of imaging studies. No complete responses were observed for patients in either treatment arm. Partial responses were observed for $31 \%$ of patients treated with sunitinib and $6 \%$ of those treated with interferon. Median PFS was 11 months for patients in the sunitinib arm and 4 months for those in the interferon arm (hazards ratio 0.42; 95\% Cl [confidence interval] 0.33-0.52). All differences were highly statistically significant. At the time of analysis, median survival was not reached 
in either arm, but a trend toward improved survival that did not meet the prespecified level of significance for interim analysis was observed (hazards ratio for death $0.64 ; 95 \% \mathrm{Cl} 0.45-0.94$; $p=0.02$ ). Subgroup analyses suggested that patients with various risk factors seemed to have similar benefits with sunitinib. When divided into good-, intermediate- or poor-risk categories according to Motzer criteria, ${ }^{3}$ patients in all 3 categories who received sunitinib fared better than those who received interferon. ${ }^{10}$ Although few patients were categorized in the poor-risk group (23 patients in the sunitinib group and 25 in the interferon group), the trend was toward improvement in median PFS (4 mo v. 1 mo, respectively) (hazards ratio $0.53 ; 95 \% \mathrm{Cl} 0.23-1.23)$. Health-related quality of life measured in this study was better in the sunitinib group than in the interferon group $(p<0.001)$.

The results of this large phase 3 trial convincingly demonstrated that sunitinib is significantly better than interferon for previously untreated patients with advanced RCC and should be considered the new standard of care for first-line treatment of this disease. Although no phase 3 study has demonstrated similar findings for patients who previously received cytokine therapy, extrapolation of the first-line findings from this large trial, along with the impressive response rates of over $40 \%$ observed in 2 phase 2 studies, suggests that sunitinib would also be a reasonable choice for patients refractory to or intolerant of cytokine therapy.

These results do not justify the use of sunitinib for patients at earlier stages of RCC, such as adjuvantly in patients at high risk for recurrence after nephrectomy. This particular patient population is the subject of multiple ongoing or planned clinical trials. Referral of patients to centres participating in such trials is strongly encouraged.

\section{Sorafenib}

Sorafenib is an oral multikinase inhibitor that was originally identified as a Raf kinase inhibitor. ${ }^{12}$ It inhibits other receptors, including VEGFR-1, VEGFR-2 and VEGFR-3.12,13 A large phase 2 randomized discontinuation trial ${ }^{14}$ reported that patients with advanced RCC who had progressed after previous systemic therapy had improved PFS, compared with patients receiving placebo. This finding sparked interest in further research for this particular patient population.

No phase 3 studies of sorafenib for patients who have received no prior systemic therapy, such as cytokine therapy, have been published. A completed phase 2 study $^{15}$ comparing sorafenib with interferon in that patient population has presented only toxicity data so far. Grade 3 or higher hematological toxicities, such as lipase elevation, increased partial thromboplastin time and hypophosphatemia, as well as grade 3 or higher nonhematological adverse events, such as handfoot skin reactions; rash and pain were observed more frequently with sorafenib than with interferon. Interferon was associated with more fatigue. Patients randomized to interferon treatment were permitted to cross over to sorafenib treatment when their disease progressed. Although the primary end point was overall survival, a predetermined stopping rule was based on PFS. The criteria for stopping this trial were met at a planned interim analysis; the trial was unblinded and patients receiving placebo were offered sorafenib. Progression-free survival was the primary end point of the trial. Overall survival, objective tumour-response rate, quality of life and adverse effects were also assessed. Until efficacy results are presented, sorafenib cannot be considered a standard of care for patients with previously untreated advanced RCC. However, the consensus of most RCC experts is that extrapolation from favourable results for previously treated patients suggests that sorafenib may be a suitable alternative to sunitinib for patients with advanced RCC and a good or an intermediate prognosis who are intolerant of or thought to be at significant risk for moderate toxicity after treatment with sunitinib. Previously untreated patients with a poor prognosis should likely be offered temsirolimus (see below) or best supportive care alone.

The Treatment Approaches in Renal Cancer Global Evaluation Trial (TARGET) ${ }^{16}$ has also recently been published in the New England Journal of Medicine. This international phase 3 study randomized 903 patients who were resistant to prior systemic therapy to oral sorafenib at a continuous dose of $400 \mathrm{mg}$ twice daily or an identical-looking placebo. The primary end point of the study was overall survival and the main secondary end point was PFS. Most patients had clear-cell histology, 51\% were in the low-risk prognostic group and $49 \%$, in 
the intermediate-risk group. Ninety-three percent of patients had had nephrectomy, and $82 \%$ cytokine therapy as their prior systemic treatment. An independent committee reviewed the data about the safety and efficacy of sorafenib. An interim analysis had been planned after about 270 patients had died. In April 2005, on the basis of the first PFS analysis by the committee, the patients were unblinded and sorafenib offered to the patients assigned to receive placebo. Because crossover could compromise the end point of survival, the trial was amended to allow a first analysis of overall survival at the start of treatment crossover in May 2005, after 220 deaths had occurred.

The median duration of treatment was 23 weeks in the sorafenib group and 12 weeks in the placebo group. Similar proportions of patients discontinued treatment because of adverse events in each group $(10 \%$ in the sorafenib group and $8 \%$ in the placebo group). Thirteen percent of patients receiving sorafenib required dose reductions and $21 \%$ of patients required dose interruptions because of adverse effects. The median duration of dose interruption was 7 days; the interruptions were mostly due to dermatological events, such as handfoot skin reactions or rash, and gastrointestinal events, such as diarrhea. Other common adverse events were fatigue, alopecia and nausea. Most adverse events were grade 1 or 2 in intensity and were self-limiting. Hypertension occurred more frequently in the sorafenib group than in the placebo group, was usually observed in the first cycle of treatment and led to permanent discontinuation in less than $1 \%$ of patients. Cardiac ischemia or infarction occurred in 12 (3\%) of 451 patients in the sorafenib group compared with 2 (less than $1 \%)$ patients treated with placebo $(p=0.01)$.

The first analysis of overall survival was done immediately before the crossover was allowed, when 220 deaths $(41 \%$ of the protocol-defined 540 deaths) had occurred. With a median follow-up of 6.6 months, the median actuarial overall survival was 14.7 months in the placebo group, but had not been reached in the sorafenib arm (hazard ratio $0.72 ; 95 \% \mathrm{Cl} 0.54-0.94 ; p=0.02$ ). When survival was assessed 6 months later, 216 of 452 patients receiving placebo had switched to sorafenib and 367 deaths had occurred. Median overall survival was 19.3 months in the sorafenib group and 15.9 months in the placebo group (hazards ratio $0.77 ; 95 \% \mathrm{Cl} 0.63-0.95)$. For both analy- ses, the $p$ value was 0.02 , which was less than the prespecified $\mathrm{O}^{\prime}$ Brien-Fleming values for statistical significance for preliminary analyses.

Results of the first analysis of PFS indicated a median PFS of 5.5 months in the sorafenib arm, compared with 2.8 months in the placebo arm (hazards ratio $0.44 ; 95 \% \mathrm{Cl} 0.35-0.55 ; p<0.001$ ). Independent reviewers assessed the best radiological response. Among the 451 patients in the sorafenib group, 1 (less than 1\%) patient had a complete response, $43(10 \%)$ patients had a partial response, and $333(74 \%)$ had stable disease. In the placebo group of 452 patients, no patients had a complete response, $8(2 \%)$ patients had a partial response and 239 (53\%) patients had stable disease. Among the 44 patients with a complete or partial response on sorafenib, the median time to response was 80 days (range 35-275 d) and the median duration of response was 182 days (range 36-378 d). To grade the response, responseevaluation criteria in solid tumours (RECIST) were used. However, most patients on the sorafenib arm with stable disease still had regression of tumours that did not meet RECIST end points for response. The utility of the criteria developed for RECIST for the evaluation of inhibitors of angiogenesis and for other more cytostatic agents that lead to prolonged stable disease and moderate tumour shrinkage have recently been challenged. ${ }^{17}$

The results of this large phase 3 trial $^{16}$ have convincingly demonstrated that sorafenib is significantly better than placebo for the treatment of patients with advanced RCC who have received prior systemic therapy and should be considered the new standard of care for this patient population. Although no phase 3 study has demonstrated similar findings for this first-line treatment, extrapolation from the TARGET trial, ${ }^{16}$ along with what many experts consider sorafenib's more favourable toxicity profile, suggest that sorafenib could also be a reasonable choice for previously untreated patients. The final results of the randomized phase 2 study will help determine the specific role of sorafenib for this patient population.

Similar to the results for sunitinib, these results do not justify the use of sorafenib for treatment of patients at an earlier stage of RCC, such as adjuvantly in patients at high risk for recurrence of the disease after nephrectomy. Referral of potential patients to centres participating in such trials should be considered. 


\section{Bevacizumab}

Although the end result remains inhibition of angiogenesis through VEGF inhibition, bevacizumab differs from agents such as sorafenib and sunitinib by its mechanism of action. Bevacizumab is a recombinant human monoclonal antibody against VEGF that binds and neutralizes all biologically active isoforms of VEGF. ${ }^{18} \mathrm{~A}$ published phase 2 trial ${ }^{19}$ randomized previously treated patients with metastatic RCC to placebo, low-dose ( $3 \mathrm{mg} / \mathrm{kg}$ ) bevacizumab or high-dose bevacizumab $(10 \mathrm{mg} / \mathrm{kg})$ intravenously every 2 weeks. Four partial responses were observed, all in the high-dose arm. The time to progression in the high-dose arm was 4.8 months, compared with 2.5 months in the placebo arm $(p<0.001)$. Treatment was well tolerated and hypertension was one of the more frequently encountered adverse events. In the high-dose arm, hypertension of any grade occurred in 36\% of patients and grade 3 hypertension, in $21 \%$ of patients. Asymptomatic proteinuria without renal dysfunction was observed in $64 \%$ of patients in the high-dose arm. All toxicities were reversible.

Given the promising data from that trial, a large intergroup phase 3 trial $^{20}$ randomizing patients to either interferon alone or interferon and highdose bevacizumab was undertaken. The study has completed accrual and results are eagerly anticipated. Until these results are released, bevacizumab cannot be recommended as a treatment option for patients with advanced RCC.

\section{The mTOR inhibitor temsirolimus}

The mTOR pathway has become a central target for cancer therapy. The mTOR protein kinase is a 289-kDa serine/threonine-specific kinase, of which the carboxyl group of the target of rapamycin (TOR) is similar to the catalytic domain of PI3-kinase (PI3K). ${ }^{21}$ mTOR primarily functions as a key controller of cell proliferation, growth and survival. TOR centrally regulates cell growth and proliferation by regulating the initiation of translation. TOR regulates the translation of ribosomal proteins, and 2 proteins in particular: p70S6K1 and 4E-BP1. Thus, the TOR pathway controls the translation of mRNA that encodes proteins that are required for $\mathrm{G} 1$ cell-cycle progression and S-phase initiation. TOR acts as a gatekeeper for cell-cycle progression, and TOR inhibition results in a pro- longed G1 phase or G1 arrest. Substantial preclinical data have established the pTEN-PI3K-Akt-mTOR pathway as a major oncogenic pathway linked to the development of some of the most common human cancers.

Temsirolimus, or CCl-779, a soluble ester analog of rapamycin, was selected for development as an anticancer agent, based on its prominent antitumour profile and favourable pharmaceutical and toxicological characteristics in preclinical studies. ${ }^{22}$ Temsirolimus was found to have improved aqueous solubility and stability over rapamycin as an anticancer agent. Temsirolimus is a specific inhibitor of mTOR kinase, a signaling protein that regulates cell growth and angiogenesis. ${ }^{23}$ Temsirolimus binds to FKBP-12, which in turn forms a complex that inhibits the mTOR pathway. ${ }^{24}$ By inhibiting mTOR signaling, temsirolimus inhibits the translation of several key proteins that regulate the cell cycle and angiogenesis. ${ }^{25}$

A phase 2, single-agent study ${ }^{26}$ was designed to evaluate the efficacy, safety and pharmacokinetics of temsirolimus for patients with advanced refractory RCC. This study was a randomized, double-blinded, multicentre trial (111 centres) of patients with cytokine-refractory metastatic RCC, including patients with poor-risk features. Patients were randomly assigned to receive $25 \mathrm{mg}, 75 \mathrm{mg}$ or $250 \mathrm{mg}$ of temsirolimus weekly as a 30-minute intravenous infusion. Patients were evaluated for tumour response, time to tumour progression, survival, and adverse events. Temsirolimus produced an objective response of $7 \%$ ( 1 complete and 7 partial responses) and minor responses in 26\% of patients. Median time to progression was 5.8 months and median survival time was 15.0 months. Within each risk group, the median survival of patients at each dose level was similar. The most frequently occurring temsirolimus-related adverse events of all grades were maculopapular rash $(76 \%)$, mucositis $(70 \%)$, asthenia $(50 \%)$ and nausea (43\%). Maculopapular rash (seen in 5 patients) was the most frequent reason for treatment discontinuation. Pneumonitis was seen in 6 patients, of whom 2 were withdrawn from the study, 2 became worse after restarting the drug and 2 had no recurrence of pneumonitis after restarting drug. The notable activity in patients with poor-prognosis features prompted a phase $3 \mathrm{trial}^{27}$ for patients with poor prognostic features.

This phase 3 study for first-line treatment of 
poor-risk patients with RCC was initiated in July 2003. Patients with advanced RRC and no prior systemic therapy were enrolled if they had 3 of 6 risk factors (the 5 Motzer criteria and more than 1 metastatic disease site). Of the 626 patients with advanced RCC and poor-risk features enrolled, $67 \%$ had prior nephrectomy. Patients were randomized $(1: 1: 1)$ to up to 18 million units interferon subcutaneously 3 times weekly (arm 1); $25 \mathrm{mg}$ temsirolimus intravenously once weekly (arm 2); or 15 mg temsirolimus intravenously weekly, plus 6 million units interferon subcutaneously 3 times weekly (arm 3). The primary study end point was overall survival, and the study was powered to compare the temsirolimus arms with the interferon-alone arm. Poor-risk features for eligibility included a minimum of 3 of the following features:

- lactate dehydrogenase greater than 1.5 times the upper limit of normal

- hemoglobin below the lower limit of normal

- corrected calcium greater than $10 \mathrm{mg} / \mathrm{dL}$

- time from diagnosis to first treatment less than 1 year

- Karnofsky performance status of 60-70

- multiple-organ sites of metastasis

The 3 most frequently occurring adverse events were asthenia (arm 1:arm 2:arm 3, 27\%:12\%:30\% of patients), anemia (24\%:21\%:39\% of patients) and dyspnea (8\%:9\%:11\% of patients). Less common adverse events included nausea (5\%:4\%:2\% of patients), vomiting (0\%:1\%:5\% of patients), hyperlipidemia ( $1 \%: 7 \%: 2 \%$ of patients), hyperglycemia ( $1 \%: 10 \%: 4 \%$ of patients) and neutropenia (8\%:3\%:14\% patients). More patients in the interferon groups discontinued treatment because of adverse events (14:7:22 patients). Single-agent temsirolimus $(n=209)$, compared with interferon $(n=207)$, significantly increased the overall survival (10.9 mo v. $7.3 \mathrm{mo} ; p=0.0069$ ) of patients with metastatic RCC and poor-risk factors. Median overall survival by treatment arm was 7.3 months (interferon), 10.9 months (temsirolimus) and 8.4 months (combination). Median PFS was 1.9 months (interferon), 3.7 months (temsirolimus) and 3.7 months (combination). Objective responses were observed in $7 \%, 9 \%$ and $11 \%$ of patients, respectively.

Single-agent temsirolimus (25 mg given intravenously weekly) significantly increased the overall survival of previously untreated patients with advanced RCC and poor-risk features, when compared with interferon, which had an acceptable safety profile. Single-agent temsirolimus can therefore be considered an acceptable standard of care for this specific population. Similar to the other agents discussed in this review, further research is necessary to expand the role of temsirolimus in early disease states and for patients with a good or an intermediate prognosis for their disease.

\section{Summary and conclusions}

The systemic therapeutic landscape for patients with advanced RCC has changed dramatically in recent years. Healthcare professionals who treat these patients have met these new challenges with great enthusiasm. Sorafenib, sunitinib and temsirolimus have all been shown to significantly alter the natural history of advanced RCC, and many more agents are currently being evaluated..$^{28}$ Trials evaluating combinations of these agents are currently underway or planned, and the optimal sequence of use of these agents is also being evaluated. However, patients, clinicians and researchers alike are all keenly aware that much more study is necessary before they will know whether these novel inhibitors of angiogenesis can provide the ultimate clinical benefit to patients diagnosed with RCC - a cure for their disease.

From the *Department of Oncology, Juravinski Cancer Centre and the †Department of Surgery (Urology), St. Joseph's Health Care, Hamilton, Ont.

This article has been peer reviewed.

Competing interessts: None declared for Dr. Kapoor. Dr. Hotte has received research grants, honoraria and speaker's fees from Bayer, Pfizer and Wyeth.

\section{References}

1. Canadian Cancer Society/National Cancer Institute of Canada. Canadian Cancer Statistics. Toronto: National Cancer Institute of Canada; 2006.

2. Bukowski RM. Immunotherapy in renal cell carcinoma. Oncology 1999;13:801-10.

3. Motzer RJ, Mazumdar M, Bacik J, et al. Survival and prognostic stratification of 670 patients with advanced renal cell carcinoma. J Clin Oncol 1999;17:2530-40.

4. Amato RJ. Chemotherapy for renal cell carcinoma. Semin Oncol 2000;27:177-86.

5. Cohen HT, McGovern FJ. Renal-cell carcinoma. N Engl J Med 2005;353:2477-90.

6. Dvorak HF. Vascular permeability factor/vascular endothelial growth factor: a critical cytokine in tumor angiogenesis and a potential target for diagnosis and therapy. J Clin Oncol 2002;20:4368-80.

7. Abrams TJ, Lee LB, Murray $\mathrm{U}$, et al. SU1 1248 inhibits KIT and platelet-derived growth factor receptor beta in preclinical models of human small cell lung cancer. Mol Cancer Ther 2003;2:471-8.

8. Mendel DB, Laird AD, Xin X, et al. In vivo antitumor activity of SU1 1248, a novel tyrosine kinase inhibitor targeting vascular endothelial growth factor and platelet-derived 
growth factor receptors: determination of a pharmacokinetic/pharmacodynamic relationship. Clin Cancer Res 2003;9:327-37.

9. O'Farrell AM, Abrams TJ, Yuen HA, et al. SU1 1248 is a novel FLT3 tyrosine kinase inhibitor with potent activity in vitro and in vivo. Blood 2003;101:3597-605.

10. Motzer RJ, Hutson TE, Tomczak $P$, et al. Sunitinib versus interferon alfa in metastatic renal-cell carcinoma. N Engl J Med 2007;356:115-24.

11. Motzer RJ, Rini BI, Bukowski RM, et al. Sunitinib in patients with metastatic renal cell carcinoma. JAMA 2006;295:2516-24.

12. Wilhelm SM, Carter C, Tang L, et al. BAY 43-9006 exhibits broad spectrum oral antitumor activity and targets the Rat/MEK/ERK pathway and receptor tyrosine kinases involved in tumor progression and angiogenesis. Cancer Res 2004;64:7099-109.

13. Carlomagno F, Anaganti S, Guida T, et al. BAY 43-9006 inhibition of oncogenic RET mutants. J Natl Cancer Inst 2006;98:326-34.

14. Ratain MJ, Eisen T, Stadler WM, et al. Phase II placebo-controlled randomized discontinuation trial of sorafenib in patients with metastatic renal cell carcinoma. J Clin Oncol 2006;24:2505-12.

15. Escudier B, Szczylik C, Demkow T, et al. Randomized phase II trial of the multikinase inhibitor sorafenib versus interferon (IFN) in treatment-naive patients with metastatic renal cell carcinoma (mRCC). [abstract]. [Ann Meet Proc]. J Clin Oncol 2006;24(Suppl 18):4501.

16. Escudier B, Eisen T, Stadler WM, et al. Sorafenib in advanced clear-cell renal-cell carcinoma. N Engl J Med 2007;356:125-34.

17. Ratain MJ, Eckhardt SG. Phase II studies of modern drugs directed against new targets: if you are fazed, too, then resist RECIST. JClin Oncol 2004;22:4442-5.

18. Presta $L G$, Chen $\mathrm{H}, \mathrm{O}^{\prime}$ Connor $\mathrm{SJ}$, et al. Humanization of an anti-vascular endothelial growth factor monoclonal antibody for the therapy of solid tumors and other disorders. Cancer Res 1997:57:4593-9.

19. Yang JC, Haworth L, Sherry RM, et al. A randomized trial of bevacizumab, an antivascular endothelial growth factor antibody, for metastatic renal cancer. N Engl J Med 2003:349:427-34.
20. Rini BI, Halabi S, Taylor J, et al. Cancer and Leukemia Group B 90206: a randomized phase III trial of interferon-alpha or interferon-alpha plus anti-vascular endothelial growth factor antibody (bevacizumab) in metastatic renal carcinoma. Clin Cancer Res 2004;10:2584-6.

21. Houghton PJ, Huang S. mTOR as a target for cancer therapy. Curr Top Microbiol Immunol 2004:279:339-59

22. Hidalgo $M$, Rowinski EK. The rapamycin-sensitive signal transduction pathway as a target for cancer therapy. Oncogene 2000;19:6680-6.

23. Schmelzle T, Hall MN. TOR, a central controller of cell growth. Cell 2000;103:253-62.

24. Skotnicki JS, Leone CL, Smith AL, et al. Design, synthesis and biological evaluation of $\mathrm{C}-42$ hydroxyesters of rapamycin: the identification of $\mathrm{CC}-779$. Clin Cancer Res 2001;7:3749S-50S.

25. Thomas GV, Tran C, Mellinghoff IK, et al. Hypoxia-inducible factor determines sensitivity to inhibitors of mTOR in kidney cancer. Nat Med 2005;12:122-7.

26. Atkins MB, Hidalgo M, Stadler WM, et al. Randomized phase II study of multiple dose levels of $\mathrm{CCl}-779$ in patients with advanced refractory renal cell carcinoma. J Clin Oncol 2004;22:909-18.

27. Hudes $G$, Carducci $M$, Tomczak $P$, et al. A phase 3, randomized 3-arm study of temsirolimus (TEMSR) or interferon-alpha (IFN) or the combination of TEMSR + IFN in the treatment of first-line, poor-risk patients with advanced renal cell carcinoma (adv RCC). [abstract]. [Ann Meet Proc]. J Clin Oncol 2006;24(Suppl 18):LBA4.

28. Morabito A, De Maio E, Di Maio M, et al. Tyrosine kinase inhibitors of vascular endothelial growth factor receptors in clinical trials: current status and future directions. Oncologist 2006;11:753-64.

Correspondence: Dr. Sébastien J. Hotte, Juravinski Cancer Centre, Department of Oncology, Division of Medical Oncology, 699 Concession St., Hamilton ON L8V 5C2; Sebastien.Hotte@hrcc.on.ca 